\title{
Progressive multifocal leukoencephalopathy: MRI findings in HIV-infected patients are closer to rituximab- than natalizumab-associated PML
}

\author{
Manel Alleg $^{1}$ (D) Morgane Solis $^{2} \cdot$ Seyyid Baloglu ${ }^{1} \cdot$ François Cotton $^{3,4} \cdot$ Philippe Kerschen $^{5} \cdot$ Bertrand Bourre $^{6}$. \\ Guido Ahle ${ }^{7}$ (D) Jean-Pierre Pruvo ${ }^{8} \cdot$ Xavier Leclerc $^{8} \cdot$ Patrick Vermersch $^{9} \cdot$ Caroline Papeix $^{10} \cdot$ Élisabeth Maillart $^{10}$. $^{1}$. \\ Caroline Houillier $^{11}$. Cécile Moluçon Chabrot ${ }^{12}$ - Béatrice Claise ${ }^{12}$ - Sandra Malak ${ }^{13}$ - Guillaume Martin-Blondel ${ }^{14,15}$. \\ Fabrice Bonneville ${ }^{16}$. Alexis Caulier ${ }^{17}$. Jean-Pierre Marolleau ${ }^{17}$ • Jérôme Tamburini Bonnefoy ${ }^{18} \cdot$ Philippe Agape $^{19}$. \\ Céline Kennel $^{20} \cdot$ Xavier Roussel $^{21}$ - Adrien Chauchet ${ }^{21} \cdot$ Jérôme De Seze $^{22}$ - Samira Fafi-Kremer ${ }^{2}$. \\ Stéphane Kremer ${ }^{1,23}$
}

Received: 25 January 2020 / Revised: 26 August 2020 / Accepted: 29 September 2020 / Published online: 6 November 2020

(C) European Society of Radiology 2020

\section{Abstract}

Objectives To compare brain MRI findings in progressive multifocal leukoencephalopathy (PML) associated to rituximab and natalizumab treatments and HIV infection.

Materials and methods In this retrospective, multicentric study, we analyzed brain MRI exams from 72 patients diagnosed with definite PML: 32 after natalizumab treatment, 20 after rituximab treatment, and 20 HIV patients. We compared T2- or FLAIRweighted images, diffusion-weighted images, T2*-weighted images, and contrast enhancement features, as well as lesion distribution, especially gray matter involvement.

Results The three PML entities affect U-fibers associated with low signal intensities on T2*-weighted sequences. Natalizumabassociated PML showed a punctuate microcystic appearance in or in the vicinity of the main PML lesions, a potential involvement of the cortex, and contrast enhancement. HIV and rituximab-associated PML showed only mild contrast enhancement,

Manel Alleg

Manel.alleg@gmail.com

1 Hôpitaux Universitaires de Strasbourg, Service d'imagerie 2, Hôpital de Hautepierre, Strasbourg, France

2 Hôpitaux universitaires de Strasbourg, Laboratoire de Virologie Médicale, Strasbourg, France

3 MRI center, Centre Hospitalier Lyon Sud, Hospices Civils de Lyon,, Lyon, France

4 Université Lyon 1, CREATIS-LRMN, CNRS/UMR/5220-INSERM U630, Villeurbanne, France

5 Centre hospitalier de Luxembourg, Luxembourg City, Luxembourg

6 Rouen University Hospital, F-76000 Rouen, France

7 Service de Neurologie, Hôpitaux Civils de Colmar, Colmar, France

8 Department of Neuroradiology, University of Lille, Inserm UMR-S 1172, CHU Lille, Lille, France

9 Univ-Lille, Inserm UMR 1172, CHU Lille, FHU Imminent, Lille, France

10 Department of Neurology, CRC-SEP, Pitié-Salpêtrière Hospital, APHP, Paris, France
11 APHP, Sorbonne Université, IHU, ICM, Service de Neurologie 2-Mazarin, Groupe Hospitalier Pitié-Salpêtrière, Paris, France

12 CHU de Clermont Ferrand, Clermont Ferrand, France

13 Service d'hématologie, Institut Curie-Saint-Cloud, Saint-Cloud, France

14 Service des Maladies Infectieuses et Tropicales, CHU de Toulouse, Toulouse, France

15 UMR INSERM/CNRS 1043, Centre de Physiopathologie Toulouse Purpan, Toulouse, France

16 Service de Neuroradiologie, CHU de Toulouse, Toulouse, France

17 Hématologie Clinique, CHU Amiens-Picardie, Amiens, France

18 Hôpital Cochin Paris, Paris, France

19 Institut de Cancérologie de l'Ouest, Saint-Herblain Nantes, France

20 CHU de Limoges, Limoges, France

21 CHU de Besançon, Besançon, France

22 Service de Neurologie, Hôpitaux Universitaires de Strasbourg Hôpital de Hautepierre, Strasbourg, France

23 Engineering Science, Computer Science and Imaging Laboratory (ICube) UMR 7357, University of Strasbourg-CNRS,

Strasbourg, France 
punctuate appearance, and cortical involvement. The CD4/CD8 ratio showed a trend to be higher in the natalizumab group, possibly mirroring a more efficient immune response.

Conclusion Imaging features of rituximab-associated PML are different from those of natalizumab-associated PML and are closer to those observed in HIV-associated PML.

Key Points

- Nowadays, PML is emerging as a complication of new effective therapies based on monoclonal antibodies.

- Natalizumab-associated PML shows more inflammatory signs, a perivascular distribution "the milky way," and more cortex involvement than rituximab- and HIV-associated PML.

- MRI differences are probably related to higher levels of immunosuppression in HIV patients and those under rituximab therapy.

Keywords Progressive multifocal leukoencephalopathy $\cdot$ Natalizumab $\cdot$ Rituximab

\begin{tabular}{ll}
\multicolumn{2}{l}{ Abbreviations } \\
AAN & American Academy of Neurology \\
AIDS & Acquired immune deficiency syndrome \\
DNA & Deoxyribonucleic acid \\
DWI & Diffusion-weighted images \\
FLAIR & Fluid-attenuated inversion recovery \\
GM & Gray matter \\
HIV & Human immunodeficiency virus \\
IRIS & Immune reconstitution inflammatory syndrome \\
JCV & JC virus \\
MRI & Magnetic resonance imaging \\
MS & Multiple sclerosis \\
PCR & Polymerase chain reaction \\
PML & Progressive multifocal leukoencephalopathy \\
SWI & Susceptibility-weighted imaging \\
WM & White matter
\end{tabular}

\section{Introduction}

Progressive multifocal leukoencephalopathy (PML) is a rare demyelinating disease of the central nervous system caused by reactivation of the JC polyomavirus (JCV). JCV is a ubiquitous polyomavirus, present in nearly $80 \%$ of the adult population [1]. Primary infection is asymptomatic and leads to viral persistence in infected hosts in different sites such as bone marrow and kidneys. JCV reactivation is triggered by a decrease in immunity [2], more particularly in cell-mediated immunity [3]. PML incidence increased notably during the 1980s with the HIV pandemic supporting the hypothesis of the essential role played by cell-mediated immunity $[1,4]$.

Nowadays, PML is emerging as a complication of new effective immunosuppressive and immunomodulatory drugs [5]. Natalizumab is used as a treatment for multiple sclerosis (MS) and is associated with an increased risk of PML, estimated at $1 / 100$ to $1 / 10,000$ based on three identified risk factors (treatment duration longer than 24 months, anti-JCV antibody index, and prior administration of immunosuppressive therapies) [6]. Anti-JCV antibody levels in serum are indeed used to stratify PML risk in anti-JCV antibody-positive MS patients [6].

Natalizumab is a monoclonal antibody targeting the alpha4 integrin that inhibits transmigration of $\mathrm{T}$ cells, including CD8 T cells, to and in the central nervous system, which can explain in part its role in PML through a decrease of immune control of JCV in the brain [1]. Two other characteristics are that natalizumab forces the migration of hematopoietic stem cells from the bone marrow in which JCV may be persistent and the upward regulation of $\mathrm{B}$ cell maturation factors that promote JCV growth [7].

Rituximab, an anti-CD20 monoclonal antibody targeting B cell activity and currently used for hematological malignancies and autoimmune disorders, is also associated with a moderately increased risk of PML estimated at 1/32,000 [8]. Rituximab acts by depleting B cells and modifying $\mathrm{T}$ cell activity, all of which supports a role of rituximab in PML development [9].

Knowledge of the MRI pattern of PML is essential when this diagnosis is suspected, especially in populations considered at risk, because the prognosis remains poor and is based on early treatment [10], which consists exclusively so far in restoring the immune system.

In the classic form widely described in the HIV population, PML manifests itself as white matter (WM) lesions affecting the U-shaped subcortical fibers with a decreased signal on T1weighted sequences and an increased signal on T2-weighted sequences. On diffusion-weighted images (DWI), the signal is generally increased with restricted diffusion on borders corresponding to active demyelination. Low signal on magnetic susceptibility sequences in U-fibers adjacent to the WM lesions of PML has been recently described and appears in relation to intracellular accumulation of iron in macrophages and glial cells during myelin degeneration [15]. Those lesions are often not enhanced and have little or no mass effect [11]. Previous studies have shown differences in the MRI pattern with natalizumab-associated PML (Table 1). Indeed, with natalizumab, there are various inflammatory signs such as contrast enhancement and small punctuations in or adjacent 
Table 1 Key MRI features of HIV-associated PML, natalizumab-associated PML and IRIS-PML according to the litterature review

\begin{tabular}{|c|c|c|c|}
\hline & $\begin{array}{l}\text { HIV-PML } \\
\text { MRI key features }\end{array}$ & $\begin{array}{l}\text { Natalizumab-PML } \\
\text { MRI key features }\end{array}$ & $\begin{array}{l}\text { IRIS-PML } \\
\text { key features }\end{array}$ \\
\hline References & {$[11,12]$} & {$[2]$} & {$[13,14]$} \\
\hline $\begin{array}{l}\text { Location and aspect on } \\
\text { T2/FLAIR }\end{array}$ & $\begin{array}{l}\text { Hypersignal } \\
\text { Subcortical, affecting U-fibers } \\
\text { Posterior fossa is frequently affected } \\
\text { especial middle cerebellar peduncles } \\
\text { Occasionally, lesions may be limited to } \\
\text { the cerebellum and/or brainstem } \\
\text { Respect of the spinal cord or optic nerves }\end{array}$ & $\begin{array}{l}\text { Hypersignal with a punctuate or microcystic } \\
\text { aspect in the vicinity of the main PML } \\
\text { lesions Subcortical, affecting U-fibers, } \\
\text { possibly the cortex and the deep GM, not } \\
\text { respecting the WM/GM border }\end{array}$ & $\begin{array}{l}\text { Aggravation with extension or } \\
\text { PML lesions or new lesion }\end{array}$ \\
\hline Edges & $\begin{array}{l}\text { Clear sharp towards GM when U-fibers are } \\
\text { involved }\end{array}$ & $\begin{array}{l}\text { Clear sharp towards GM and ill-defined to- } \\
\text { wards WM }\end{array}$ & \\
\hline Mass effect & Absent & Absent & Present with brain edema \\
\hline Contrast enhancement & Rare & Frequent, punctuate, or linear & Frequent \\
\hline Diffusion & $\begin{array}{l}\text { Hyperintense with a peripheral rim in } \\
\text { restriction of diffusion }\end{array}$ & Hyperintense & Hyperintense \\
\hline
\end{tabular}

to lesions with an increased signal on T2-weighted images and a perivascular distribution called "the milky way" [2, 16, 17]. The lesions have ill-defined boundaries towards WM and sharp boundaries towards gray matter (GM), with cortical GM more frequently involved [18]. A hypothesis to explain these results would be the difference in the level of immunosuppression induced by HIV and natalizumab.

In this study, we first aimed to compare the MRI presentation of rituximab-associated PML to natalizumab or HIVassociated PML, and also analyze biological parameters to assess if the differences in MRI could be the consequence of different immunosuppression levels.

\section{Materials and methods}

\section{Patients}

This retrospective study was approved by our institutional review board.

Inclusion criteria were (1) a "definite" PML diagnosis according to the American Academy of Neurology criteria [19] including clinical and imaging-compatible features and detection of JCV DNA in the cerebrospinal fluid or in brain tissue by polymerase chain reaction (PCR) or immunohistochemistry; (2) HIV-infected patients or patients treated with immunomodulatory or immunosuppressive drugs such as natalizumab or rituximab, possibly in association with other drugs and whatever the initial illness; and (3) consent was provided for in the hospital's charter. Exclusion criteria were patients diagnosed with PML from other causes than HIV infection or associated with other drugs than natalizumab or rituximab and those under 18 years of age. The occurrence of Immune Reconstitution Inflammatory Syndrome (IRIS) or an opportunistic infection was not a cause for exclusion.
Classical demographic data such as gender, age at the time of diagnosis, and underlying illness were collected, as well as CD4 and CD8 numbers. Between 2008 and 2018, 87 patients from 16 centers in France and Luxembourg were retrospectively identified (Fig. 1). Of these 87 patients, 72 fulfilled all the inclusion criteria and were therefore included: 32 patients had relapsing-remitting $\mathrm{MS}$ and were treated with natalizumab, 20 patients were treated with rituximab (16 had a hematopoietic malignancy, 2 an autoimmune disorder, and 2 an immune deficiency), and 20 patients had an HIV infection.

\section{Imaging}

All patients underwent a multisequence MRI protocol. Due to the study's multicenter design, the explorations were carried out with diverging sequences, magnetic fields strength, acquisition parameters and parameters related to spatial resolution. We chose to define the first brain MRI performed at suspicion of PML as the reference examination for all comparisons. All MRIs were unblinded and reviewed by a junior radiologist (a trainee) with the assistance of an experienced senior neuroradiologist.

To compare PML lesions, we described the location (frontal lobe, parietal lobe, occipital lobe, temporal lobe, basal ganglia, supra- or infratentorial or both), involvement of cortex and/or deep GM, distribution (unilobar involving a single lobe, multilobar involving more than one contiguous lobe, widespread involving more than two contiguous lobes or noncontiguous lobes or bilaterality), boundaries (sharp towards WM and GM, ill-defined towards WM and GM), lesion characteristics (aspect on T2-weighted images defined as homogeneous or microcystic corresponding to small lesions in or adjacent to the main PML lesions with an increased signal called the milky way; the presence or absence of a hyposignal on $\mathrm{T} 2 * / \mathrm{SWI} / \mathrm{SWAN}$-weighted images, signal on DWI, and 
Fig. 1 Flow chart illustrating the patient selection and inclusion process. $P M L=$ progressive multifocal leukoencephalopathy, $A A N=$ American Academy of Neurology

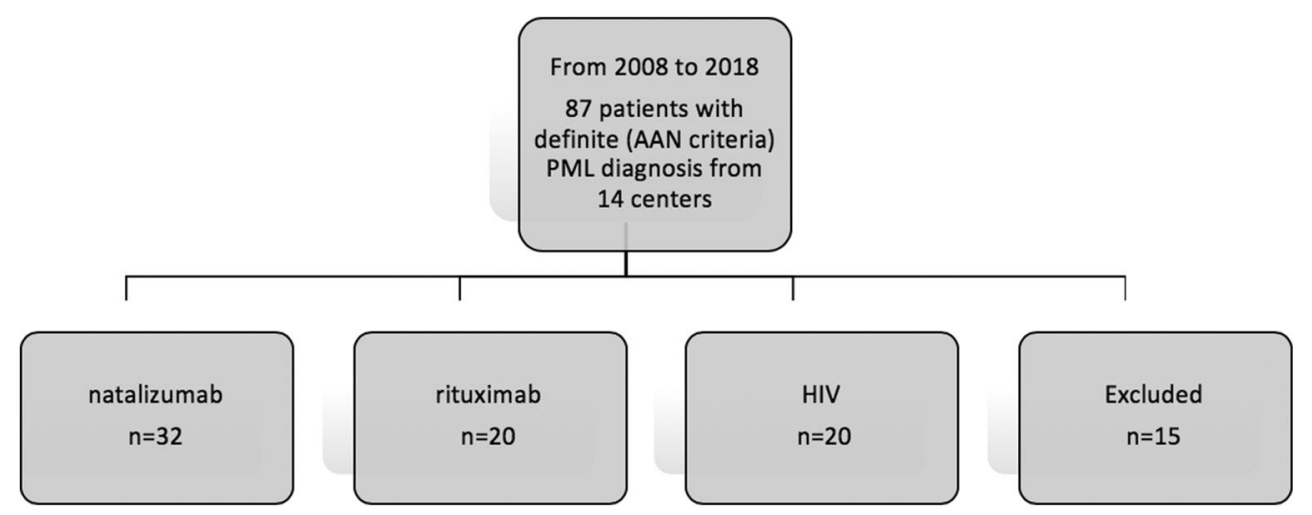

the presence or absence of a rim of diffusion restriction); contrast enhancement (defined as absent, surrounding, homogeneous punctuate or adjacent).

\section{Statistical analysis}

Statistical analysis was performed using $\mathrm{R}$ software, version 3.1, via the GMRC Shiny Stat application of the Strasbourg University Hospital (2017) and using the XLSTAT software in its Addinsoft 2016 version. We compared the MRI findings between the three groups using the chi-square test and between the NAT/RITUX, NAT/HIV, and RITUX/HIV groups using the chi-square test with Holm correction. We compared biological data between the three groups using the Shapiro test and between the NAT/RITUX, NAT/HIV, and RITUX/HIV groups using the Student $t$ test with Holm correction.

\section{Results}

\section{Demographic data}

Median patient age (for all groups) was 49.0 years (range, 2676). In the patient groups, the median age was 42.62 years for the natalizumab group (range, 26-53), 61.5 years for the rituximab group (range, 40-76) and 46.9 for the HIV group (range, 26-64). Forty men (55.6\%) and 32 women $(44.4 \%)$ were included in the study: 14 men (43.8\%) and 18 women $(56.2 \%)$ in the natalizumab group, 10 men and 10 women (50\% each) in the rituximab group, and 16 men (80\%) and 4 women $(20 \%)$ in the HIV group.

\section{Imaging results}

The imaging results are summarized in Table 2 and Table 3.

\section{Location of PML lesions}

There was a statistically significant difference in supratentorial involvement between the three groups $\mathbf{7 8 . 1 \%}$ in the natalizumab group, $85 \%$ in the rituximab group, and $40 \%$ in the HIV group ( $p=0.003)$, especially when comparing the natalizumab group vs the HIV group $(p=0.0269)$ and the rituximab group vs the HIV group $(p=0.0269)$ with fewer supratentorial lesion locations in cases of PML occurring in an AIDS context. Concerning GM involvement, there was a significant difference between the three groups for the cortex $(p<0.001)$ and for the basal ganglia $(p=0.025)$. Post hoc analysis was only contributive for cortex involvement and showed a difference between the natalizumab group vs the HIV/rituximab groups with more cortex involvement in cases of PML that occurred under natalizumab treatment than the other two groups $(p=0.0036$ for natalizumab vs rituximab and $p=0.0084$ for natalizumab vs HIV).

\section{Aspect of PML lesions}

Analysis of the edges towards both WM and GM (Fig. 2) showed significant differences between groups. Indeed, lesions were more frequently clearly defined towards GM and ill-defined towards WM in the natalizumab group with no significant difference between the rituximab and HIV groups.

The milky way aspect on T2- or FLAIR-weighted images (Fig. 2) was significantly different between the three groups $(p=0.02)$ and more often seen in the natalizumab group vs the rituximab group $(51.6 \%$ for natalizumab and $5 \%$ for rituximab $[p=0.005])$. On DWI, the vast majority of PML lesions had an increased signal (90.9\% for natalizumab, $89.5 \%$ for rituximab and $100 \%$ for HIV). We isolated cases with a peripheral signal in restriction of diffusion (corresponding to a decrease of the signal on ADC mapping), called the demyelination front. For this parameter, we observed a significant difference between natalizumab/rituximab $(22.7 \%$ for natalizumab, $63.2 \%$ for rituximab $[p=0.0426])$ and natalizumab/HIV (22.7\% for natalizumab, $84.2 \%$ for HIV, $[p<0.001])$ but none between the rituximab and HIV groups. (Fig. 3). Lesions with a peripheral contrast enhancement showed a significant difference between the three groups $(p=0.003)$ and tended 
Table 2 Summary of imaging results

\begin{tabular}{|c|c|c|c|}
\hline MRI findings & Natalizumab & Rituximab & HIV \\
\hline Localization & $N=32$ & $N=20$ & $N=20$ \\
\hline Supratentorial & $78.1 \%(25 / 32)$ & $85 \%(17 / 20)$ & $40 \%(B / 20)$ \\
\hline Infratentorial & $6.2 \%(2 / 32)$ & $10 \%(2 / 20)$ & $20 \%(4 / 20)$ \\
\hline Both & $15.6 \%(5 / 32)$ & $5 \%(1 / 20)$ & $40 \%(8 / 20)$ \\
\hline Bilaterality & $62.5 \%(20 / 32)$ & $75 \%(15 / 20)$ & $55 \%(11 / 20)$ \\
\hline Frontal & $75 \%(24 / 32)$ & $75 \%(15 / 20)$ & $70 \%(14 / 20)$ \\
\hline Temporal & $21.9 \%(7 / 32)$ & $35 \%(7 / 20)$ & $55 \%(11 / 20)$ \\
\hline Parietal & $43.8 \%(14 / 32)$ & $55 \%(11 / 20)$ & $45 \%(9 / 20)$ \\
\hline Occipital & $46.9 \%(15 / 32)$ & $40 \%(8 / 20)$ & $45 \%(9 / 20)$ \\
\hline Unilobal & $28.1 \%(9 / 32)$ & $15 \%(3 / 20)$ & $15 \%(3 / 20)$ \\
\hline Multilobar & $15.6 \%(5 / 32)$ & $10 \%(2 / 20)$ & $15 \%(3 / 20)$ \\
\hline Widespread & $56.2 \%(18 / 32)$ & $5 \%(1 / 20)$ & $70 \%(14 / 20)$ \\
\hline Cortex reached & $59.4 \%(19 / 32)$ & $10 \%(2 / 20)$ & $15 \%(3 / 20)$ \\
\hline Basal ganglia reached & $25 \%(8 / 32)$ & $5 \%(1 / 20)$ & $40 \%(8 / 20)$ \\
\hline Thalamus & $21.9 \%(7 / 32)$ & $0 \%(0 / 20)$ & $30 \%(6 / 20)$ \\
\hline Corpus callosum & $9.4 \%(3 / 32)$ & $20 \%(4 / 20)$ & $35 \%(7 / 20)$ \\
\hline Inner capsule & $12.5 \%(4 / 32)$ & $20 \%(4 / 20)$ & $45 \%(9 / 20)$ \\
\hline Cerebellum & $18.8 \%(6 / 32)$ & $10 \%(2 / 20)$ & $55 \%(11 / 20)$ \\
\hline Brainstem & $21.9 \%(7 / 32)$ & $10 \%(2 / 20)$ & $50 \%(10 / 20)$ \\
\hline Edges & $N=32$ & $N=20$ & $N=20$ \\
\hline III-defined towards WM & $100 \%(32 / 32)$ & $35 \%(7 / 20)$ & $96 \%[19]$ \\
\hline Sharp towards WM & $0 \%(0 / 32)$ & $60 \%(12 / 20)$ & $5 \%[1]$ \\
\hline III-defined towards GM & $6.2 \%(2 / 32)$ & $15 \%(3 / 20)$ & $45 \%[9]$ \\
\hline Sharp towards GM & $93.8 \%(30 / 32)$ & $85 \%(17 / 20)$ & $55 \%[14]$ \\
\hline T2/FLAIR & $N=32$ & $N=20$ & $N=20$ \\
\hline Homogeneous hypersignal & $96.8 \%(30 / 31)$ & $100 \%(20 / 20)$ & $100 \%[20]$ \\
\hline Microcystic hypersignal & $51.6 \%(16 / 31)$ & $5 \%(1 / 20)$ & $30 \%[7]$ \\
\hline T2*/SWAN/SWI & $N=16$ & $N=17$ & $N=18$ \\
\hline Hypointense signal & $62.5 \%(10 / 16)$ & $47.1 \%(8 / 17)$ & $36.9 \%[7]$ \\
\hline DWI & $N=22$ & $N=19$ & $N=19$ \\
\hline Hypersignal & $100 \%(22 / 22)$ & $94.7 \%(18 / 19)$ & $100 \%[19]$ \\
\hline Rim of demyelination & $22.7 \%(5 / 22)$ & $63.2 \%(12 / 19)$ & $84.2 \%[18]$ \\
\hline $\mathrm{ADC}$ & $N=22$ & $N=19$ & $N=19$ \\
\hline Unchanged & $9.1 \%(2 / 22)$ & $10.5 \%(2 / 19)$ & $0 \%(0)$ \\
\hline High & $90.9 \%(20 / 22)$ & $89.5 \%(17 / 19)$ & $100 \%[19]$ \\
\hline Enhancement & $N=31$ & $N=20$ & $N=20$ \\
\hline None & $38.7 \%(12 / 31)$ & $55 \%(11 / 20)$ & $80 \%[18]$ \\
\hline Surrounding & $19.4 \%(6 / 31)$ & $45 \%(9 / 20)$ & $10 \%[2]$ \\
\hline Homogeneous & $6.5 \%(2 / 31)$ & $0 \%(0 / 20)$ & $0 \%(0)$ \\
\hline Punctuate & $51.6 \%(16 / 31)$ & $10 \%(2 / 20)$ & $20 \%[4]$ \\
\hline Remote & $38.7 \%(12 / 31)$ & $0 \%(0 / 20)$ & $5 \%[1]$ \\
\hline
\end{tabular}

to predominate in the rituximab group $(45 \%$ in the rituximab group vs $19.4 \%$ in the natalizumab group and $10 \%$ in the HIV group). Lesions with micronodular enhancement were significantly different between the three groups ( $p=$ $0.003)$, with more cases in the natalizumab group versus the rituximab group ( $p=0.0187)$. There was no difference between natalizumab and HIV groups even if there were more lesions of this type in the natalizumab group $(51.6 \%$ vs $20 \%$ in the HIV group). Finally, for distant enhancement, there was a difference between the three groups $(p<0.001)$, specifically between natalizumab and rituximab $(p=0.0134)$ and the natalizumab and HIV $(p=$ 0.0358 ) groups, with more of this type of enhancement in the natalizumab group (Fig. 4). Non-enhanced lesions were 
Table 3 Comparison between groups

\begin{tabular}{|c|c|c|c|c|}
\hline MRI findings & $\begin{array}{l}\text { Comparison of the three } \\
\text { groups }\end{array}$ & $\begin{array}{l}\text { Natalizumab vs } \\
\text { rituximab }\end{array}$ & $\begin{array}{l}\text { Natalizumab vs } \\
\text { HIV }\end{array}$ & $\begin{array}{l}\text { Rituximab vs } \\
\text { HIV }\end{array}$ \\
\hline \multicolumn{5}{|l|}{ Localization } \\
\hline Supratentorial & 0.003 & 0.8023 & 0.0269 & 0.0269 \\
\hline Cortex reached & $<0.001$ & 0.0036 & 0.0084 & 1 \\
\hline $\begin{array}{l}\text { Basal ganglia } \\
\text { reached }\end{array}$ & 0.025 & 0.2788 & 0.4058 & 0.0693 \\
\hline Thalamus & 0.022 & 0.1342 & 0.742 & 0.0805 \\
\hline Inner capsule & 0.034 & 0.7382 & 0.0637 & 0.3538 \\
\hline Cerebellum & 0.02 & 0.6485 & 0.0321 & 0.0208 \\
\hline Brainstem & 0.012 & 0.4688 & 0.1439 & 0.0472 \\
\hline \multicolumn{5}{|l|}{ Edges } \\
\hline $\begin{array}{l}\text { III-defined } \\
\text { towards WM }\end{array}$ & $<0.001$ & $<0.001$ & 0.8107 & $<0.001$ \\
\hline $\begin{array}{l}\text { Sharp towards } \\
\text { WM }\end{array}$ & $<0.001$ & $<0.001$ & 0.8107 & 0.0015 \\
\hline $\begin{array}{l}\text { III-defined } \\
\text { towards GM }\end{array}$ & 0.003 & 0.577 & 0.0087 & 0.169 \\
\hline Sharp towards GM & 0.003 & 0.577 & 0.0087 & 0.169 \\
\hline \multicolumn{5}{|l|}{ T2/FLAIR } \\
\hline $\begin{array}{c}\text { Microcystic } \\
\text { hypersignal } \\
\text { T2*/SWAN/SWI }\end{array}$ & 0.02 & 0.005 & 0.218 & 0.192 \\
\hline $\begin{array}{l}\text { Hypointense } \\
\text { signal }\end{array}$ & 0.381 & & & \\
\hline \multicolumn{5}{|l|}{ DWI } \\
\hline $\begin{array}{l}\text { Rim of } \\
\text { demyelination }\end{array}$ & $<0.001$ & 0.0426 & $<0.001$ & 0.2691 \\
\hline \multicolumn{5}{|l|}{ Enhancement } \\
\hline None & 0.015 & 0.3935 & 0.0276 & 0.3538 \\
\hline Surrounding & 0.003 & 0.1988 & 0.6153 & 0.1008 \\
\hline Homogeneous & 0.501 & & & \\
\hline Punctuate & 0.003 & 0.0187 & 0.0991 & 0.6579 \\
\hline Remote & $<0.001$ & 0.0134 & 0.0358 & 1 \\
\hline
\end{tabular}

significantly different between the three groups ( $p=$ 0.015), higher in the HIV groups with $80 \%$.

\section{Biological results}

CD4/CD8 data were available for 10 patients in the natalizumab group, 12 in the rituximab group, and 15 in the HIV group (Fig. 5). The mean CD4/CD8 ratio was 1.95 (95\% confidence interval [CI95], 1.44-2.46) in the natalizumab group, 1.09 (CI95, 0.55-1.64) in the rituximab group, and 0.12 (CI95, 0.04-0.19) in the HIV group. Statistical analysis showed that the CD4/CD8 ratio was much lower in the HIV group compared to the natalizumab and the rituximab groups (Student $t$-test with Holm correction, $p<0.001$ and $p=0.001$, respectively) and was lower in the rituximab group compared to the natalizumab group $(p=0.001)$.

\section{Discussion}

This study aims to describe the MRI characteristics of PML associated with rituximab and natalizumab and in HIV infection while comparing imaging findings with the level of immunosuppression. We therefore compared CD4/CD8 ratios and found lower ratios in HIV-related PML compared to other groups, as well as a lower CD4/CD8 ratio in the rituximab group compared to the natalizumab group. This suggests that immunosuppression in rituximab is not as severe as in HIVPM but still stronger than in natalizumab-PML.

One of the characteristics of PML under natalizumab described in previous studies $[2,22]$ is the description of inflammatory signs such as microcystic lesions on T2-weighted images giving a Milky Way appearance [20, 23]. We found significantly more Milky Way lesions in the natalizumab group compared to the rituximab group but no significant 


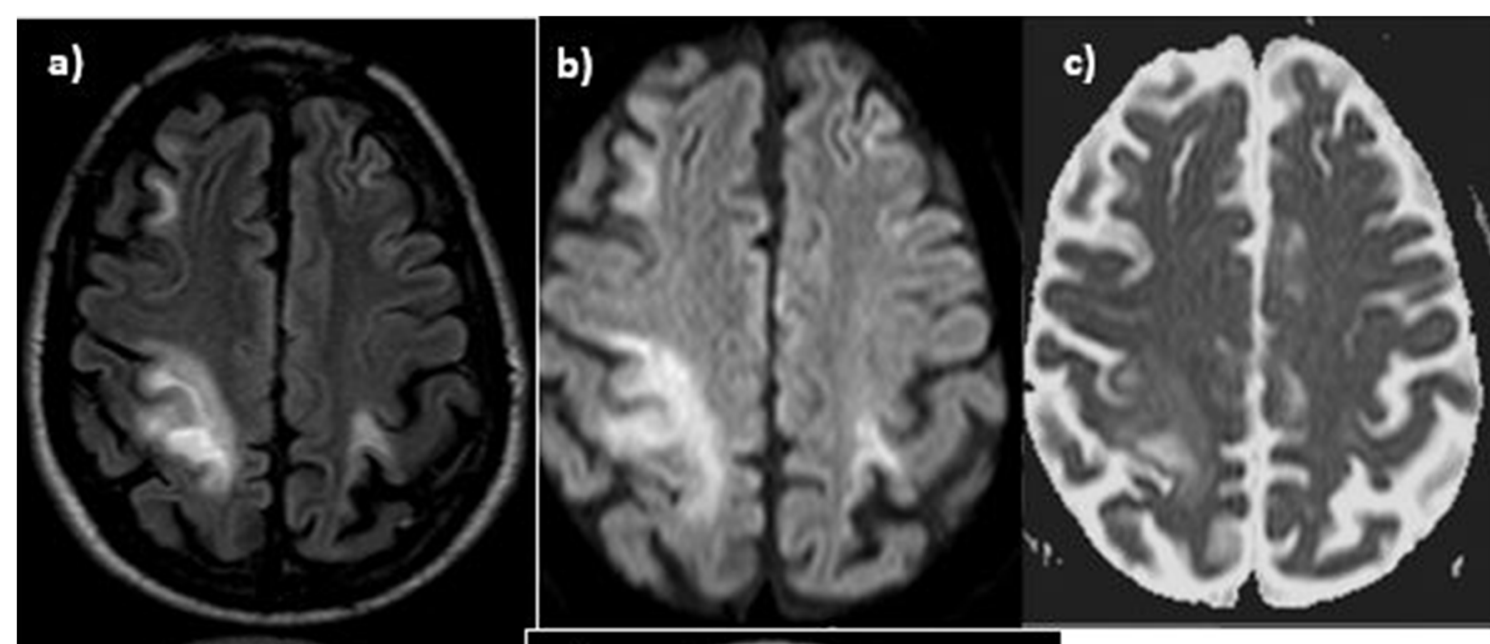

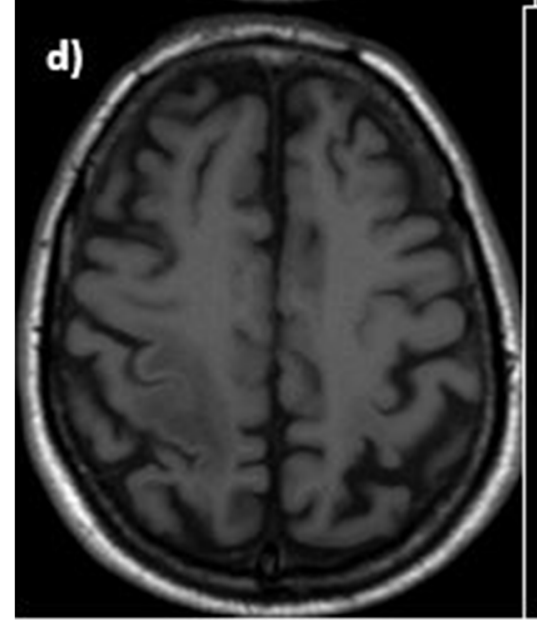

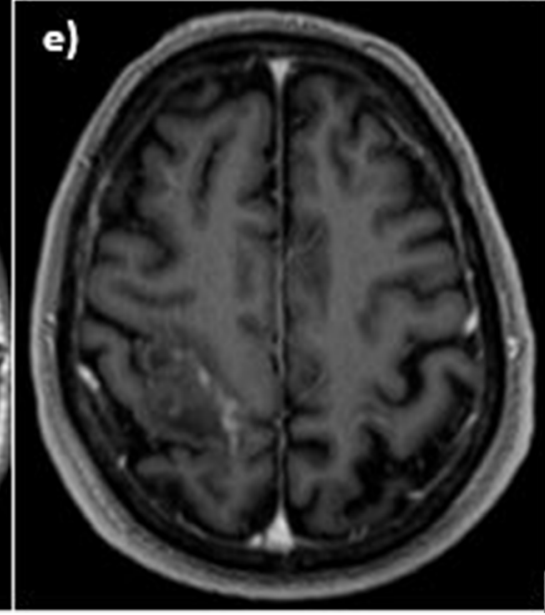

Fig. 2 Brain MRI from a patient with rituximab-associated PML. On FLAIR-weighted images (a), we observe several demyelinating supratentorial and juxtacortical lesions, bilateral and asymmetric. b Diffusion-weighted images and (c) ADC mapping show an increased

difference between the natalizumab and HIV groups, even if they tended to predominate in the natalizumab group.

The other "inflammatory" sign usually attributed to natalizumab-PML is contrast enhancement, estimated at 35 to $40 \%$ of patients in the literature [2]. In the present study, it accounted for $61.3 \%$ of the natalizumab group. A difference was observed between the three groups, especially when the enhancement was micronodular, similar to the Milky Way perivascular distribution predominating in the natalizumab group compared to the rituximab and HIV groups.

By inducing a lower degree of immunosuppression compared to HIV, natalizumab-associated PML has more inflammatory signs. This could be due to the mode of action of this drug, which is a more selective immunomodulating agent [17].

We found more supratentorial involvement in drug-related PML than in the HIV-associated form where the brainstem and cerebellum are more likely to be affected. Cortical involvement and the typical aspect of the edges (sharp towards GM and ill-defined towards WM) are found preferentially in the natalizumab group, as also found in previous studies [18, signal on diffusion with no rim of restriction. In (d) T1 without contrast and (e) T1 after intravenous injection of contrast, we observe a peripheral enhancement of the main lesion

22]. There is no obvious explanation for these characteristics in relation to a difference in the level of immunosuppression.

The proportion of hyposignal seen on $\mathrm{T} 2{ }^{*}$ - or SWANweighted images at the cortical-subcortical junction adjacent to PML lesions (Fig. 6) did not differ between PML groups, in agreement with other studies [15, 21, 24]; this sign seems nonspecific in PML for the underlying cause of immunosuppression. A demyelination front on diffusion-weighted images is often found with PML in the HIV group and in the rituximab group but more rarely in the natalizumab group. If the demyelination front is related to the active nature of the disease, we can hypothesize that the PML associated with rituximab is more like HIV-PML than natalizumab-PML, possibly due to closer immunosuppression levels [25].

\section{Limitations}

The main limitations of this study are its retrospective and unblinded design as well as the limited number of patients analyzed with complete clinical, biological, and available 

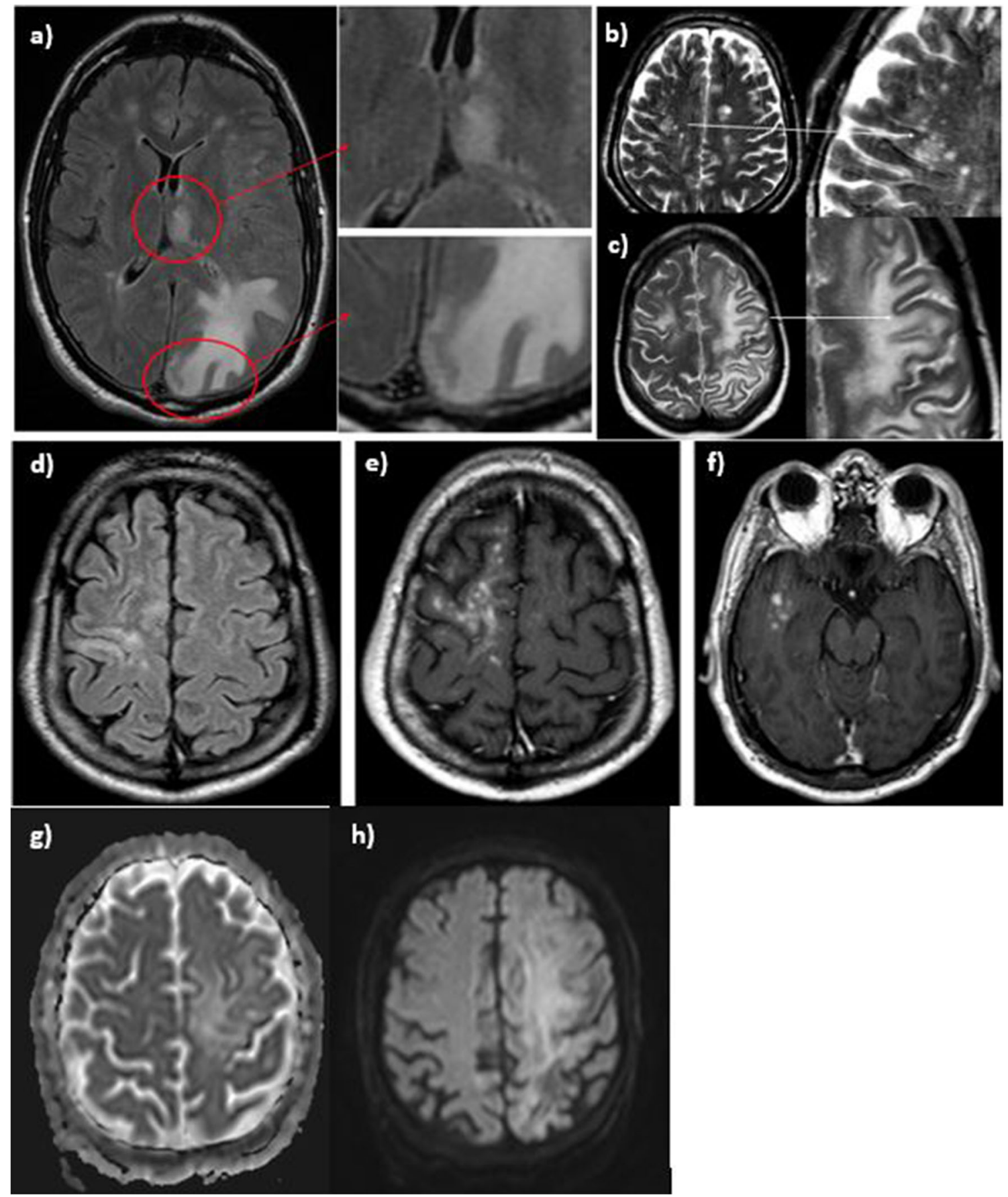

Fig. 3 In several cases of natalizumab-associated PML showing in (a) on FLAIR-weighted images, we can see GM and especially cortical involvement of the left parietal lobe adjacent to the WM lesion. The left thalamus is also the site of a demyelinating lesion. In $\mathbf{b}$, we observe on T2-weighted images the Milky Way aspect with microcystic lesions with an increased signal. In c, clear sharp edges towards GM and ill-defined edges towards

imaging data. This is explained by the relative rarity of PML even in large referral centers. Secondly, due to the multicenter design of the study, the explorations were performed on different MRIs, both $1.5 \mathrm{~T}$ and $3 \mathrm{~T}$ and with nonstandardized protocols, and there were some inevitable variations in MRI acquisition parameters between patients [26].
WM of the demyelinating lesions. In $\mathbf{d}, \mathbf{e}$, and $\mathbf{f}$, brain MRI from a patient with natalizumab-associated PML showing in (d) a demyelinating right frontal lesion on FLAIR-weighted image with (e) contrast micronodular enhancement in the lesion and (f) remotely in the right temporal lobe. Finally, we can see an increased signal on diffusion-weighted images (h) with no rim in restriction on ADC mapping (g)

Regarding the demographic data, we note that the age of the patients differs according to the group, with a tendency to younger patients in HIV and natalizumab groups. This may constitute a selection bias and is explained by the epidemiological data of the pathology studied, with MS and HIV affecting younger people compared to hematological malignancies. 
Fig. 4 An example of a HIV-

PML case with an infratentorial right cerebellar demyelinating lesion on $\mathbf{a}$. In $\mathbf{b}$, no contrast enhancement of this lesion.

Diffusion-weighted images and ADC mapping of a patient showing an increased signal on diffusion (c) with a rim of demyelination in restriction of diffusion (d)
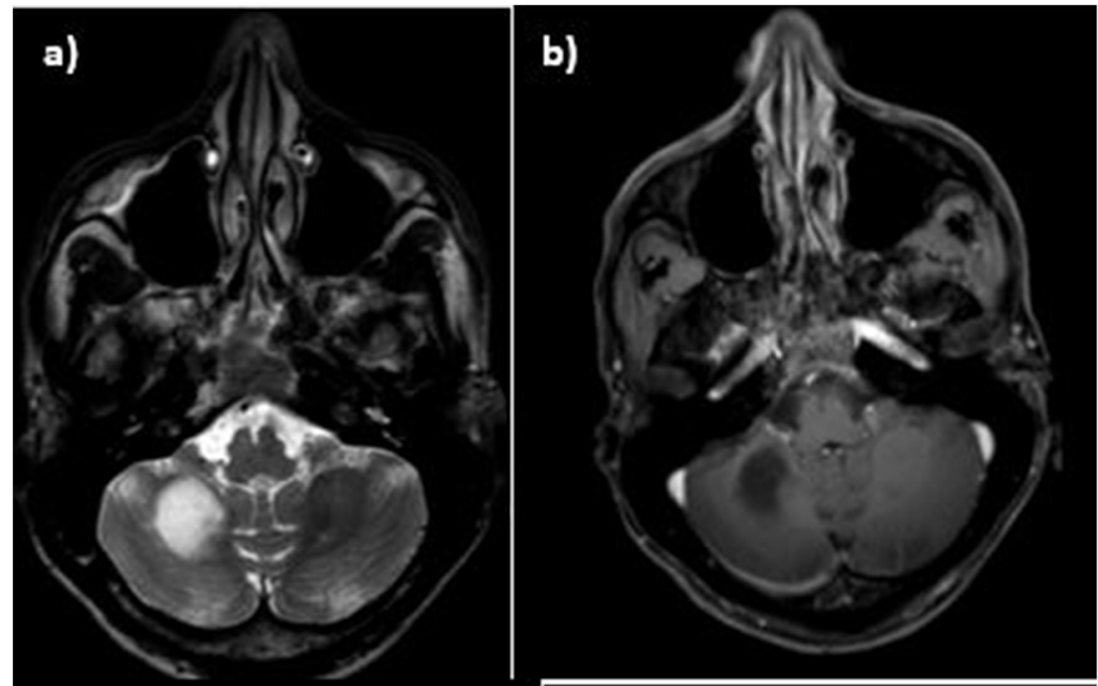

c)

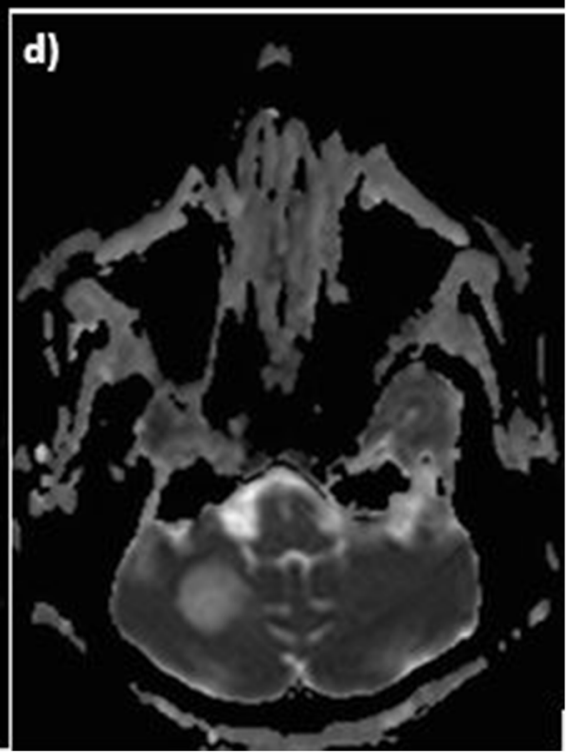

MRI interpretation was made difficult by the coexistence of inflammatory lesions in the natalizumab group related to MS as well as by the coexistence of opportunistic infections (notably cerebral toxoplasmosis for three patients in the HIV group) and the occurrence of immune reconstitution inflammatory syndrome (in three
Fig. 5 Box diagram of the extent of the $\mathrm{CD} 4 / \mathrm{CD} 8$ ratio of patients by group

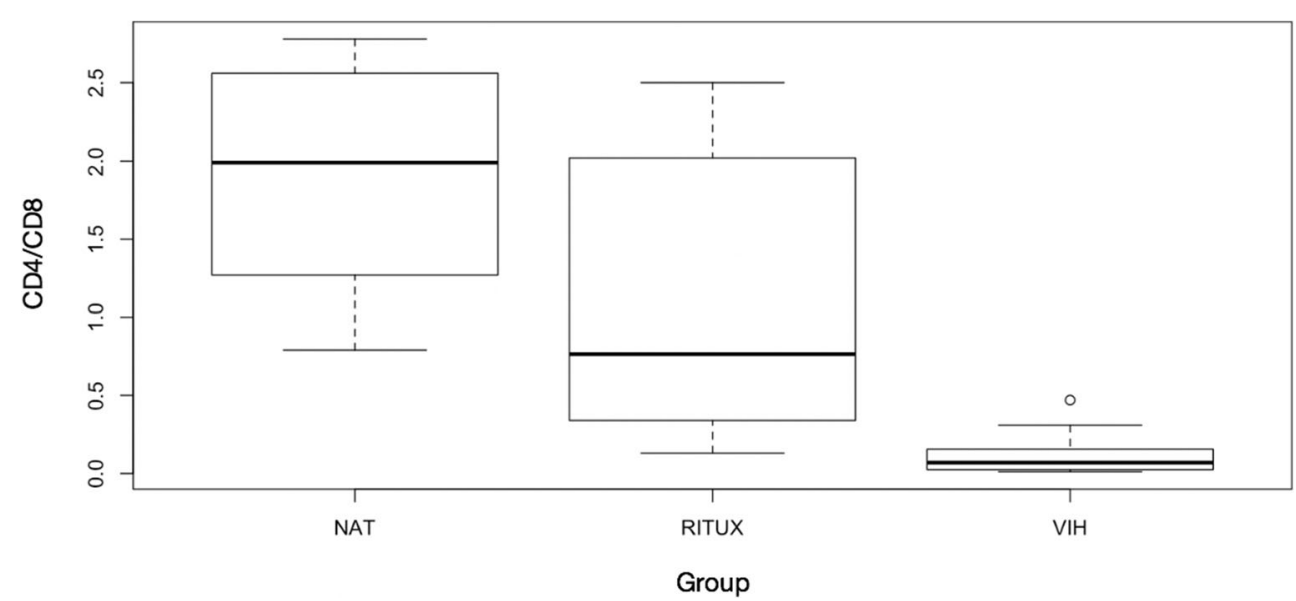


Fig. $6 \mathrm{~T} 2 *$ - and susceptibilityweighted images from patients diagnosed with PML showing a linear hyposignal on corticalsubcortical junction adjacent to PML demyelinating lesions
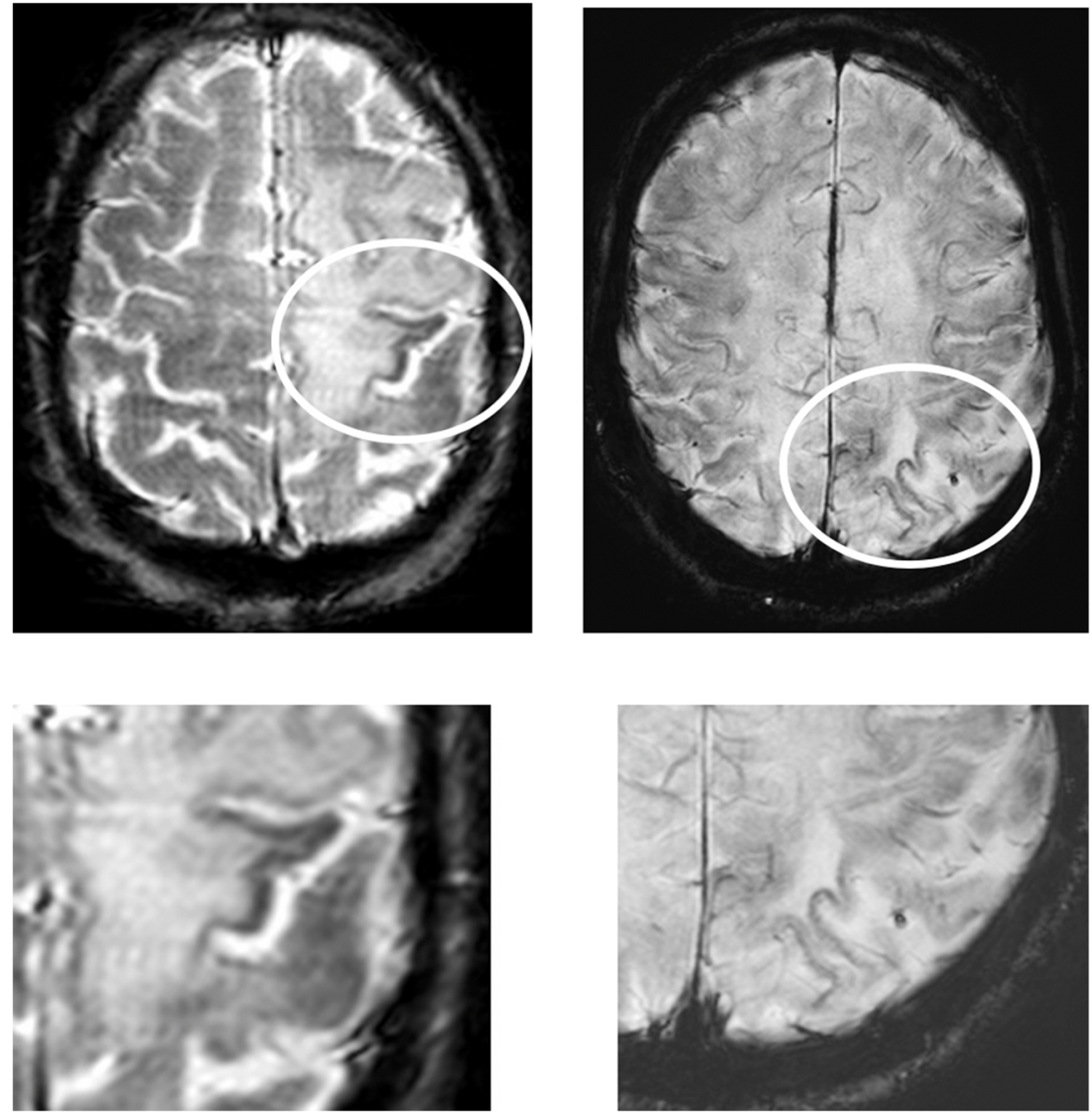

patients in the HIV group after initiation of antiretroviral therapy). Concerning inclusion criteria, the vast majority of patients in the natalizumab group had already received immunosuppressive treatments before natalizumab treatment was initiated, as had those in the rituximab group, who often had several treatments acting on immunity before or at the same time as rituximab. However, in the present study, we did not make any distinction in the groups according to these possible treatments. Their impact on the MRI pattern of PML lesions is therefore not excluded. Two other factors may have come into play in the MRI pattern of PML under rituximab that we did not consider: first, the initial disease of the patient, which could be responsible for the development of PML, especially in the case of hematopoietic malignancies [27]. Secondly, the rigorous MRI follow-up of patients in the natalizumab group made it possible to detect PML at an earlier stage than in the HIV and rituximab groups.

Adequate assessment of the quantitative and functional aspects of the global immune response is still a challenge in immunocompromised patients. In HIV-infected patients, the $\mathrm{CD} 4 / \mathrm{CD} 8$ ratio is considered to be a good marker of the remaining immune response but may not be as relevant in other immunosuppressed groups such as patients treated with natalizumab or rituximab, irrespective of the underlying disease. The CD4/CD8 ratio does not incorporate the full level of immunosuppression - due to the diversity and complexity of the immune system; in our study, it indirectly and partially reflects the patient's immune status.

\section{Conclusion}

Imaging features of rituximab-associated PML generally look more similar to those of the classically described PML with HIV than those related to natalizumab. Those similarities may be explained by a higher level of immunosuppression in HIV patients and patients treated with rituximab compared to patients treated with natalizumab.

Acknowledgments French Network for Ocular and CNS Lymphoma (LOC) and Nord-Est Neuro-Oncoly (NENO) Organisation.

Funding The authors state that this work has not received any funding.

\section{Compliance with ethical standards}

Guarantor The scientific guarantor of this publication is Stephane Kremer.

Conflict of interest The authors of this manuscript declare no relationships with any companies whose products or services may be related to the subject matter of the article. 
Statistics and biometry Nicolas Tuzin kindly provided statistical advice for this manuscript.

Informed consent Written informed consent was not required for this study because patient consent was provided for in the hospital's charter.

Ethical approval Institutional Review Board approval was obtained.

\section{Methodology}

- prospective

- observational

- multicenter study

\section{References}

1. Misbah SA (2017) Progressive multi-focal leucoencephalopathy driven from rarity to clinical mainstream by iatrogenic immunodeficiency. Clin Exp Immunol 188(3):342-352. https://doi.org/10. 1111/cei.12948

2. Wattjes MP, Richert ND, Killestein J et al (2013) The chameleon of neuroinflammation: magnetic resonance imaging characteristics of natalizumab-associated progressive multifocal leukoencephalopathy. Mult Scler 19(14):1826-1840. https://doi.org/10.1177/ 1352458513510224

3. Bauer J, Gold R, Adams O, Lassmann H (2015) Progressive multifocal leukoencephalopathy and immune reconstitution inflammatory syndrome (IRIS). Acta Neuropathol 130(6):751-764. https:// doi.org/10.1007/s00401-015-1471-7

4. De Gascun CF, Carr MJ (2013) Human polyomavirus reactivation: disease pathogenesis and treatment approaches. Clin Dev Immunol 2013:373579. https://doi.org/10.1155/2013/373579

5. Gorelik L, Lerner M, Bixler S et al (2010) Anti-JC virus antibodies: implications for PML risk stratification. Ann Neurol 68(3):295303. https://doi.org/10.1002/ana.22128

6. McGuigan C, Craner M, Guadagno J et al (2016) Stratification and monitoring of natalizumab-associated progressive multifocal leukoencephalopathy risk: recommendations from an expert group. J Neurol Neurosurg Psychiatry 87(2):117-125. https://doi.org/10. 1136/jnnp-2015-311100

7. Major EO, Yousry TA, Clifford DB (2018) Pathogenesis of progressive multifocal leukoencephalopathy and risks associated with treatments for multiple sclerosis: a decade of lessons learned. Lancet Neurol 17(5):467-480. https://doi.org/10.1016/S14744422(18)30040-1

8. Bohra C, Sokol L, Dalia S (2017) Progressive multifocal leukoencephalopathy and monoclonal antibodies: a review. Cancer Control 24(4):1073274817729901. https://doi.org/10. $1177 / 1073274817729901$

9. Kelly D, Monaghan B, McMahon E et al (2016) Progressive multifocal leukoencephalopathy secondary to rituximab-induced immunosuppression and the presence of John Cunningham virus: a case report and literature review. Radiol Case Rep 11(3):251-254. Published 2016 Jul 18. https://doi.org/10.1016/j.radcr.2016.06.003

10. Dong-Si T, Richman S, Wattjes MP et al (2014) Outcome and survival of asymptomatic PML in natalizumab-treated MS patients. Ann Clin Transl Neurol 1(10):755-764. https://doi.org/10.1002/ acn3.114

11. Shah R, Bag AK, Chapman PR, Curé JK (2010) Imaging manifestations of progressive multifocal leukoencephalopathy. Clin Radiol 65(6):431-439. https://doi.org/10.1016/j.crad.2010.03.001

12. Horger M, Beschorner R, Beck R et al (2012) Common and uncommon imaging findings in progressive multifocal leukoencephalopathy (PML) with differential diagnostic considerations. Clin Neurol Neurosurg 114(8):1123-1130. https:// doi.org/10.1016/j.clineuro.2012.06.018

13. Yousry TA, Pelletier D, Cadavid D et al (2012) Magnetic resonance imaging pattern in natalizumab-associated progressive multifocal leukoencephalopathy. Ann Neurol 72(5):779-787. https://doi.org/ 10.1002/ana.23676

14. Fournier A, Martin-Blondel G, Lechapt-Zalcman E et al (2017) Immune reconstitution inflammatory syndrome unmasking or worsening AIDS-related progressive multifocal leukoencephalopathy: a literature review. Front Immunol 8:577. Published 2017 May 23. https:// doi.org/10.3389/fimmu.2017.00577

15. Miyagawa M, Maeda M, Umino M et al (2014) Low signal intensity in U-fiber identified by susceptibility-weighted imaging in two cases of progressive multifocal leukoencephalopathy. J Neurol Sci 344(1-2):198-202. https://doi.org/10.1016/j.jns.2014.06.018

16. Clifford DB, De Luca A, Simpson DM, Arendt G, Giovannoni G, Nath A (2010) Natalizumabassociated progressive multifocal leukoencephalopathy in patients with multiple sclerosis: lessons from 28 cases. Lancet Neurol 9(4):438-446. https://doi.org/10. 1016/S1474-4422(10)70028-4

17. Wattjes MP, Wijburg MT, van Eijk J et al (2018) Inflammatory natalizumab-associated PML: baseline characteristics, lesion evolution and relation with PML-IRIS. J Neurol Neurosurg Psychiatry 89(5):535-541. https://doi.org/10.1136/jnnp-2017-316886

18. Wattjes MP, Vennegoor A, Steenwijk MD et al (2015) MRI pattern in asymptomatic natalizumab-associated PML. J Neurol Neurosurg Psychiatry 86(7):793-798. https://doi.org/10.1136/jnnp-2014308630

19. Berger JR, Aksamit AJ, Clifford DB et al (2013) PML diagnostic criteria: consensus statement from the AAN Neuroinfectious Disease Section. Neurology 80(15):1430-1438. https://doi.org/10. 1212/WNL.0b013e31828c2fa1

20. Wattjes MP, Verhoeff L, Zentjens W et al (2013) Punctate lesion pattern suggestive of perivascular inflammation in acute natalizumab-associated progressive multifocal leukoencephalopathy: productive JC virus infection or preclinical PML-IRIS manifestation? J Neurol Neurosurg Psychiatry 84(10):1176-1177. https://doi.org/10. 1136/jnnp-2013-304986

21. Hodel J, Outteryck O, Verclytte S et al (2015) Brain magnetic susceptibility changes in patients with natalizumab-associated progressive multifocal leukoencephalopathy. AJNR Am J Neuroradiol 36(12):2296-2302. https://doi.org/10.3174/ajnr.A4436

22. Honce JM, Nagae L, Nyberg E (2015) Neuroimaging of natalizumab complications in multiple sclerosis: PML and other associated entities. Mult Scler Int 2015:809252. https://doi.org/ $10.1155 / 2015 / 809252$

23. Hodel J, Darchis C, Outteryck O et al (2016) Punctate pattern: a promising imaging marker for the diagnosis of natalizumabassociated PML. Neurology 86(16):1516-1523. https://doi.org/10. 1212/WNL.0000000000002586

24. Thurnher MM, Boban J, Rieger A, Gelpi E (2019) Susceptibility-weighted MR imaging hypointense rim in progressive multifocal Leukoencephalopathy: the end point of neuroinflammation and a potential outcome predictor. AJNR Am J Neuroradiol 40(6):994-1000. https://doi.org/10.3174/ ajnr.A6072

25. Bergui M, Bradac GB, Oguz KK et al (2004) Progressive multifocal leukoencephalopathy: diffusion-weighted imaging and pathological correlations. Neuroradiology 46(1):22-25. https://doi.org/10. 1007/s00234-003-1115-9

26. Cotton F, Kremer S, Hannoun S, Vukusic S, Dousset V (2015) Imaging working Group of the Observatoire Français de la Sclérose en plaques. OFSEP, a nationwide cohort of people with multiple sclerosis: consensus minimal MRI protocol. J Neuroradiol 42(3):133-140. https://doi.org/10.1016/j.neurad.2014.12.001 
27. Focosi D, Tuccori M, Maggi F (2019) Progressive multifocal leukoencephalopathy and anti-CD20 monoclonal antibodies: what do we know after 20 years of rituximab. Rev Med Virol 29(6): e2077. https://doi.org/10.1002/rmv.2077
Publisher's note Springer Nature remains neutral with regard to jurisdictional claims in published maps and institutional affiliations. 